\title{
Stimulus and Temporal Cues in Classical Conditioning
}

\author{
Kimberly Kirkpatrick and Russell M. Church \\ Brown University
}

\begin{abstract}
In 2 experiments, separate groups of rats were given stimulus conditioning, temporal conditioning, untreated control and (in Experiment 2) learned irrelevance control procedures, followed by a compound with both stimulus and temporal cues. Stimulus conditioning consisted of a random 15-s duration conditioned stimulus (CS) followed by food; temporal conditioning consisted of food-food intervals of fixed $90 \mathrm{~s}$ (Experiment 1) or fixed $75+$ random $15 \mathrm{~s}$ (Experiment 2). The stimulus group abruptly increased responding after $\mathrm{CS}$ onset, and the temporal group gradually increased responding over the food-food interval. When the food-food interval was fixed $90 \mathrm{~s}$, the temporal cue exerted stronger control in the compound, whereas when the food-food interval was fixed 75 + random $15 \mathrm{~s}$, the stimulus cue exerted stronger control. The strength of conditioning, temporal gradients of responding, and cue competition effects appear to reflect simultaneous timing of multiple intervals.
\end{abstract}

A common view of classical conditioning is that an association is formed between the conditioned stimulus (CS) and the unconditioned stimulus (US). In associative theories of conditioning (e.g., Mackintosh, 1975; Pearce \& Hall, 1980; Rescorla \& Wagner, 1972), strength accrues to stimuli that are present at the time of US occurrence, and it accrues uniformly over the duration of a stimulus. Although associative theories can account for many of the phenomena of conditioning such as acquisition, extinction, blocking, and overshadowing, they do not assign any role of temporal content in conditioning, nor do they attempt to account for any changes in response strength over the duration of the stimulus.

There are a number of well-established facts of conditioning that indicate that temporal variables are important contributors to the conditioning process: (a) CS-US interval, trace interval, and intertrial interval durations all affect the acquisition of conditioned responses; and (b) a fixed time between the CS onset and US occurrence produces response timing relative to CS onset (inhibition of delay), and a fixed time between successive USs produces response timing relative to the time of the occurrence of the last US (temporal conditioning). To accommodate these phenomena, theories of conditioning need to include a representation of time since an event, such as CS onset or the prior US delivery.

Real-time models of conditioning (e.g., Blazis, Desmond, Moore, \& Berthier, 1986; Sutton \& Barto, 1981, 1990) have integrated a representation of time with an associative mechanism. In these models, the perception of the CS changes over its time-course so that it is possible to discriminate different times within the CS. As a result of the

Kimberly Kirkpatrick and Russell M. Church, Department of Psychology, Brown University.

Correspondence concerning this article should be addressed to Kimberly Kirkpatrick, Department of Psychology, Box 1853, Brown University, Providence, Rhode Island 02912. Electronic mail may be sent to Kim_Kirkpatrick@brown.edu. incorporation of a timing mechanism, the real-time models are able to account for the increasing likelihood of responding as a function of time since CS onset. They can also accurately reproduce many of the effects of temporal variables on conditioning, such as differences in performance due to the CS-US interval or trace interval.

Despite the recent growth of interest in the role of temporal intervals in conditioning (Cooper, 1991; Gallistel \& Gibbon, 2000; Holder \& Roberts, 1985; Holland, 1998; Kehoe, Graham-Clarke, \& Schreurs, 1989; Savastano \& Miller, 1998), there is a paucity of evidence as to how stimulus and temporal content contribute to the conditioning process. For example, it is possible that conditioning involves two processes (Desmond \& Moore, 1988; Gibbon \& Balsam, 1981): (a) learning the relationship between a CS and a US and (b) learning the temporal interval between CS onset and US occurrence. On the other hand, conditioning may involve the storage of many different features of a stimulus, including its temporal relationship with the US, spatial location, intensity, and modality (Savastano \& Miller, 1998); or, perhaps conditioning occurs through a single process that encodes the strength of association of a particular stimulus, with differential weighting over the time-course of the stimulus, as in the real-time models.

The purpose of the present study was to assess the role of stimulus and temporal factors in the conditioning process. In an initial phase, different distributions of time intervals between events were used to produce either stimulus conditioning to the CS or temporal conditioning to the food-food interval. Acquisition to the stimulus and temporal cues was examined when the two cues were delivered separately to assess differences in learning. Then, in order to determine the relative contribution of stimulus and temporal cues to the conditioning process, the two groups of rats were transferred to a compound conditioning procedure in which both the CS and the time since the prior food delivery were related to the time of the upcoming food delivery.

The design of the present study is similar to an earlier report by Williams and LoLordo (1995). They discovered 
that pretraining with a temporal cue blocked later acquisition to a stimulus cue, but pretraining with a stimulus cue did not block acquisition to a temporal cue in a compound in which both cues were present. Rats were given one trial per day of exposure to a footshock US that was delivered $1,200 \mathrm{~s}$ after the rats were placed into the experimental apparatus (the temporal cue). Conditioned freezing was measured as the conditioned response. In Experiment 1, a paired group received a 30-s CS immediately prior to US delivery, an unpaired group received a 30-s CS that began $90 \mathrm{~s}$ into the session, and a US only group did not receive any CSs. There was substantial acquisition of conditioned freezing during a period from 1,140 to $1,170 \mathrm{~s}$ (prior to CS onset in the paired group) in all three groups, indicating that the CS did not interfere with temporal conditioning. All of the rats were then given a paired procedure in which the 30-s CS preceded the US. Conditioned freezing during the CS was attenuated in the unpaired and US only groups, in contrast to the paired group. Therefore, pretraining with a temporal cue blocked later acquisition of responding to the CS. Although the Williams and LoLordo study used different procedures (aversive vs. appetitive), different stimuli, and a different measure of conditioned responding, we entertained the possibility that pretraining with the temporal cue might interfere with stimulus conditioning in the present compound conditioning procedure.

\section{Experiment 1}

Three procedures were used: stimulus conditioning, temporal conditioning, and compound conditioning. In the stimulus conditioning procedure, the CS was the only cue that was related to the occurrence of food. In the temporal conditioning procedure, the time from the prior food delivery was the only cue that was related to the time of occurrence of the next food. ${ }^{1}$ In the compound conditioning procedure, both the occurrence of the CS and the time since the prior food delivery were related to the upcoming food delivery, so that both stimulus and temporal conditioning could occur.

Presumably, the stimulus conditioning procedure should result in responding that is equally high at all times during the $\mathrm{CS}$, and the temporal conditioning procedure should result in an increase in response rate over the duration of the food-food interval. Both of these effects may be present in the compound. Prior training with the stimulus or temporal conditioning procedure, or both, may affect the relative control by the stimulus and temporal cues in the compound.

\section{Method}

\section{Subjects}

A total of 24 male Sprague-Dawley rats (Rattus norvegicus; Charles River CD, Kingston, NY), age 61 days at the beginning of the experiment, were housed individually in a colony room on a 12:12-hr light-dark cycle (lights off at 8:45 a.m.). Dim red lights provided illumination in the colony room and the testing room. The rats were fed a total daily ration of $15 \mathrm{~g}$, consisting of $45-\mathrm{mg}$ Noyes pellets (Lancaster, NH; Improved Formula A) that were delivered during the experimental session and additional FormuLab 5008 food (PMI Nutrition International, Brentwood, MO) that was given in the home cage shortly following the daily sessions. Water was available ad libitum in both the home cages and experimental chambers.

\section{Apparatus}

A total of 12 chambers $(25 \times 30 \times 30 \mathrm{~cm})$, each located inside of a ventilated, noise-attenuating box $(74 \times 38 \times 60 \mathrm{~cm})$, comprised the experimental apparatus. Each chamber was equipped with a food cup, a water bottle, and a speaker. A magazine pellet dispenser (Model ENV-203, Med Associates, St. Albans, VT) delivered 45-mg Noyes (Improved Formula A) pellets into the food cup. Each head entry into the food cup was transduced by a LED-photocell. The water bottle was mounted outside the chamber; water was available through a tube that protruded through a hole in the back wall of the chamber. The speaker for delivering white noise was situated above and to the left of the water tube. Two Gateway 486 DX2/66 computers running the Med-PC Medstate Notation Version 2.0 (Tatham \& Zurn, 1989) controlled experimental events and recorded the time at which events occurred with 10 -ms resolution.

\section{Stimuli and Responses}

The CS was a 70-dB white noise that was generated by an audio amplifier (ANL-926, Med Associates, St. Albans, VT). The rise and fall time for the onset and termination of the white noise was $10 \mathrm{~ms}$. The US was a single $45-\mathrm{mg}$ Noyes food pellet that was dropped into the food cup. The CR (and UR) was the entry of the rat's head into the food cup, a goal-tracking response that is a measure of appetitive conditioning (Bouton \& Nelson, 1998; Brown, Hemmes, deVaca, \& Pagano, 1993; Delamater, 1995; Holland, 1998; Rescorla, 1997).

\section{Procedure}

The 24 rats were randomly divided into three groups $(n=8)$ : a stimulus group, a temporal group, and a control group. The three groups were trained for two phases, lasting six sessions each. The rats were tested in two squads of 12 rats each. Testing occurred daily during 2-hr sessions that began at 9:15 a.m. and 11:30 a.m.

Pretraining (Sessions 1-6). During pretraining, the stimulus group received a stimulus conditioning procedure; the temporal group received a temporal conditioning procedure; and the rats in the control group were placed in the experimental chambers, but they did not receive any stimuli or reinforcers. The specific procedures are diagrammed in Figure 1. Each procedure is characterized by two time-event lines, one for the onset and termination of white noise CS and one for food delivery.

The stimulus conditioning procedure consisted of a random 15-s white noise, with food occurring at the time of noise termination. ${ }^{2}$

\footnotetext{
${ }^{1}$ Food delivery was chosen for the temporal conditioning procedure because we wanted to uncouple control by the stimulus and temporal cues. If CS onset had a temporal relationship with food delivery, then there would have been some relationship of the CS with food, either in the form of an actual pairing of the CS with food or as a remembered trace of the CS, as in trace conditioning.

${ }^{2}$ The random intervals were determined by exponential distributions, with the probability function $(1 / b) \mathrm{e}^{-t / b}$, where $b$ is the mean of the exponential distribution and $t$ is the time since an event
} 


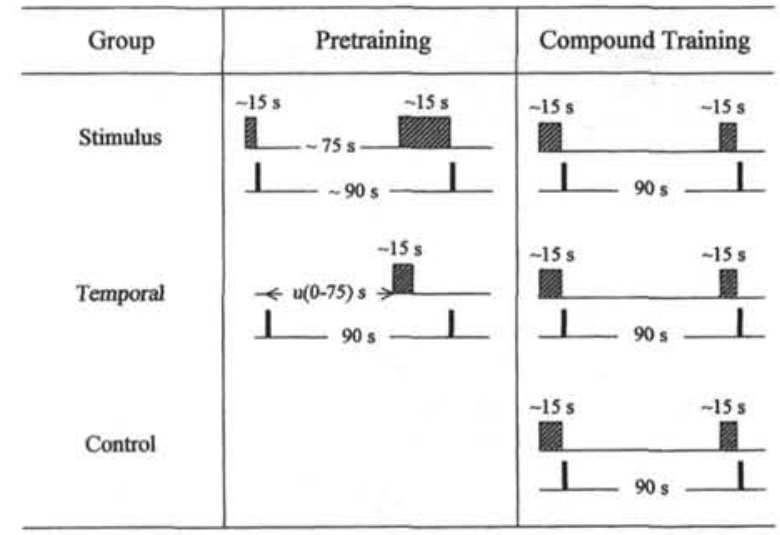

Figure 1. The schedules for delivery of the conditioned and unconditioned stimulus to the stimulus group, temporal group, and control group during the pretraining and compound training phases of Experiment 1 . The $u(0-75)$ designates a random value from a uniform distribution with a range from 0 to $75 \mathrm{~s}$. The $\sim$ designates a random value from an exponential waiting time distribution.

The passage of time since CS onset did not predict the time of food delivery, but CS onset did signal a shorter average time remaining until reinforcement. Variable duration CSs have been reported to produce robust conditioning (Davis, McIntire, \& Cohen, 1969; Kamin, 1960; Kirkpatrick \& Church, 1998; Libby \& Church, 1975) even though temporal predictability is removed. The interval between the termination of the previous noise (and delivery of the food) and the onset of the next noise was random $75 \mathrm{~s}$, so that the interval between successive food deliveries was random $90 \mathrm{~s}$. Approximately 79 food-food cycles were delivered in each session.

The temporal conditioning procedure consisted of a fixed $90-\mathrm{s}$ food-food interval. There was a random 15 -s noise that was delivered at a random time within the food-food interval. The time of noise onset was randomly chosen from a uniform (u) distribution ranging from 0 to $75 \mathrm{~s}: \mathrm{u}(0-75 \mathrm{~s})$. If the sum of the duration of the noise and the duration of the prenoise interval exceeded $90 \mathrm{~s}$, then both were resampled. The noise always occurred once during the food-food interval and usually terminated before the time of food delivery, although occasionally the noise terminated at the same time as food delivery. In all, 79 food-food cycles were delivered in each session.

Compound training (Sessions 7-12). Compound training consisted of the delivery of a compound conditioning procedure to all three groups of rats (Figure 1). The procedure consisted of a random 15-s noise, with food occurring at the time of noise termination. The food-food interval was fixed $90 \mathrm{~s}$. The time of noise onset, relative to the previous food delivery, varied inversely

(Evans, Hastings, \& Peacock, 1993). The intervals were calculated from $-b \ln (R)$, where $R$ is a uniformly distributed random number between 0 and 1 , and $\ln$ is the natural logarithm. The standard deviation of the exponential distribution is equal to the mean and the hazard function is constant at the level of $1 / b$. For an exponential distribution with a mean of $15 \mathrm{~s}$, the expected range of times encompassing $99 \%$ of the distribution would be $0.1-79.5 \mathrm{~s}$, the median of the distribution would be $10.4 \mathrm{~s}$, and the standard deviation would be $15 \mathrm{~s}$. The probability of delivering a time less than the mean of the distribution is approximately 2 times the probability of delivering a time greater than the mean. with noise duration. Because the noise always occurred at the end of the fixed duration food-food interval and because the noise was random in duration, the duration of the noise could not exceed $90 \mathrm{~s}$. If the sampled noise duration exceeded $90 \mathrm{~s}$, an exceedingly improbable event, the sample was discarded and a new sample was drawn. A total of 79 food-food cycles were delivered in each session.

\section{Data Analysis}

The time of occurrence of each head entry into the food cup (each time the photobeam was interrupted), the time of each food reinforcement and the time of onset and termination of each white noise presentation were recorded. Several measures of performance were calculated.

Discrimination ratios. Discrimination ratios (DRs) were calculated separately for control by the stimulus and temporal cues. For the assessment of control by the noise CS (the stimulus DR), the number of responses in two 2-s windows were counted for each noise occurrence. Window 1 was a 2 -s interval immediately preceding noise onset (\#before) and Window 2 was a 2-s interval immediately following noise onset (\#after). These short intervals of time were used to assess the effect of noise onset on response rate in a manner that was largely independent of the passage of time.

For the assessment of control by the passage of time since the food (the temporal DR), the number of responses were counted in two windows: $20-22 \mathrm{~s}$ after the previous food (\#early) and 73-75 s after the previous food (\#late). The rats characteristically produced high rates of food cup behavior during the first 10 to $15 \mathrm{~s}$ of the food-food interval, perhaps because they were consuming the recently delivered food pellet. This initial bout of head entry in the food cup was completed by $20 \mathrm{~s}$ in all 24 rats. The interval from 73-75 $\mathrm{s}$ was chosen because it was a time late in the food-food interval that was prior to noise onset in the compound conditioning procedure on the majority $(2 / 3)$ of occurrences. The temporal DR was not calculated if the noise was present during either window.

The number of responses in the two windows entered into the calculation of DRs. The stimulus DR was \#after/(\#after + \#before). The temporal DR was \#late/(\#late + \#early). The DRs could range from 0 to 1 , with .5 indicating that the number of responses in the two windows were equal. If both windows contained 0 responses, the DR was set to .5.

Each session was divided into five blocks of intervals, and a mean stimulus DR and temporal DR were calculated in each block. There were approximately 16 trials in each block (range $=13$ to 18 ).

Local response rate. Local response rates were determined relative to three events: CS onset, CS termination, and food delivery. Calculations of the number of responses $\left(n_{\mathrm{r}}\right)$ and the number of opportunities to respond $\left(n_{\mathrm{o}}\right)$ were conducted in each 1-s interval between an event and food delivery. Because the intervals between events and food delivery were sometimes random, the number of opportunities could vary. Local rate, expressed as responses per minute, was then defined in each 1-s interval as 60 $\left(n_{\mathrm{T}} / n_{\mathrm{o}}\right)$. For the analysis of timing from the irrelevant CS-onset and CS-termination events in Figures 3 and 7, response rates were also calculated relative to a pseudoevent to obtain a baseline estimate of responding. In Experiment 1, there was a single pseudoevent in each food-food cycle that was uniformly distributed between 0 and $90 \mathrm{~s}$. In Experiment 2, there was a random 90-s interval between successive pseudoevents, and there were no restrictions on the number or time of occurrence in the food-food cycle. Response rates relative to the pseudoevent were determined for each rat in the same manner as for the actual events. 

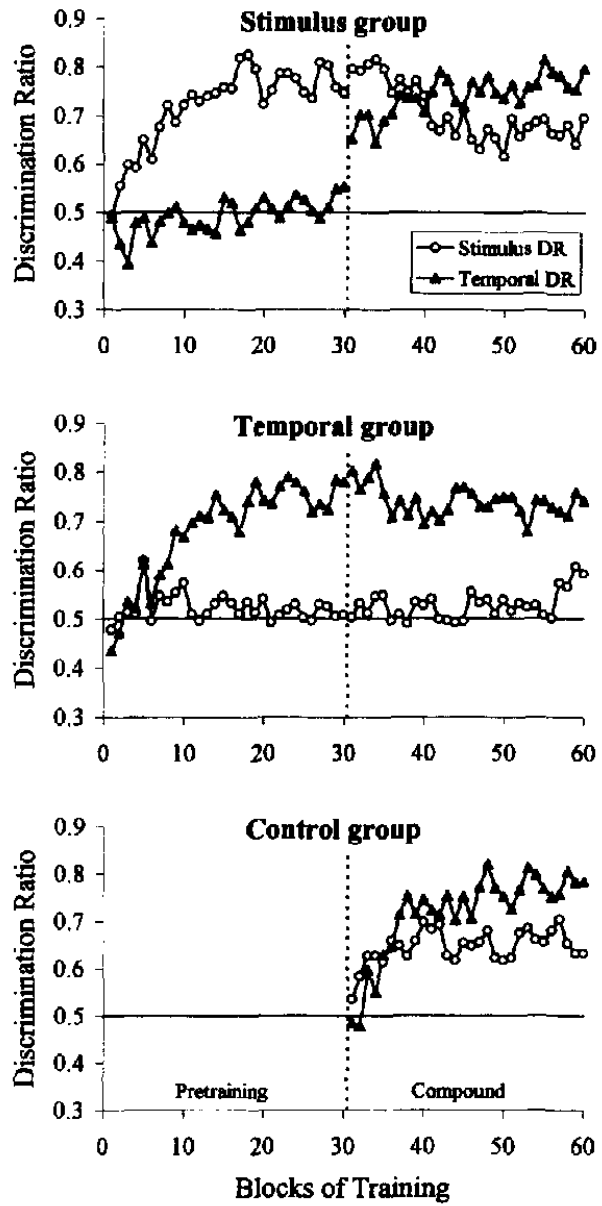

Figure 2. Acquisition of stimulus or temporal discrimination ratios (DRs) in the stimulus group (top panel), temporal group (middle panel), and control group (bottom panel) during pretraining (Blocks 1-30) and compound training (Blocks 31-60) in Experiment 1 . The strength of the stimulus DR is a measure of the degree of control by the conditioned stimulus, and the strength of the temporal DR is measure of the degree of control by the time since food. The chance level of 0.5 is indicated by the solid line.

\section{Results}

\section{Pretraining}

Discrimination ratios. The stimulus and temporal DRs produced by the stimulus group (top panel) and the temporal group (middle panel) over the course of pretraining (Blocks 21-30) are shown in Figure 2. There were no data for the control group (bottom panel) because these rats did not receive any stimuli or reinforcers during pretraining.

The acquisition of control by the relevant cue reached similar asymptotic levels in the stimulus and temporal groups, $t(14)=0.4$. Over Blocks $21-30$ of pretraining, the stimulus group demonstrated significantly greater control by the stimulus cue than by the temporal cue, $t(14)=8.0, p<$ .001 ; the temporal DRs were not significantly different from $.5(M=.51), t(7)=0.5$. The temporal group demonstrated greater control by the temporal cue than by the stimulus cue, $t(14)=-9.0, p<.001$; the stimulus DRs were not significantly different from $.5(M=.51), t(7)=1.5$.

Local response rate functions. The local response rate functions were examined for differential effects of the procedures on the time of occurrence of CRs, for both the relevant and irrelevant cues. Figure 3 displays the local response rate in 1-s intervals as a function of time relative to CS onset, CS termination, or food delivery over Sessions 4-6 of pretraining. In each panel, the event (e.g., CS onset) occurred at $0 \mathrm{~s}$, times prior to the event are shown as negative numbers, and times after the event are shown as positive numbers.

Control by the stimulus cue in the stimulus group (top-left panel, Figure 3) was exhibited as a sharp increase in responding after $\mathrm{CS}$ onset, followed by a decline in response rate until approximately $15 \mathrm{~s}$ after CS onset, and then followed by a relatively steady rate of responding from 16-45 s after CS onset. Control by the random 90-s food-food interval in the stimulus group was assessed by examining response rates as a function of time since the prior food delivery (top-right panel, Figure 3 ). This function was obtained by determining the response times relative to the time of the prior food delivery, up until the time of noise onset, so that any responding could not be due to the
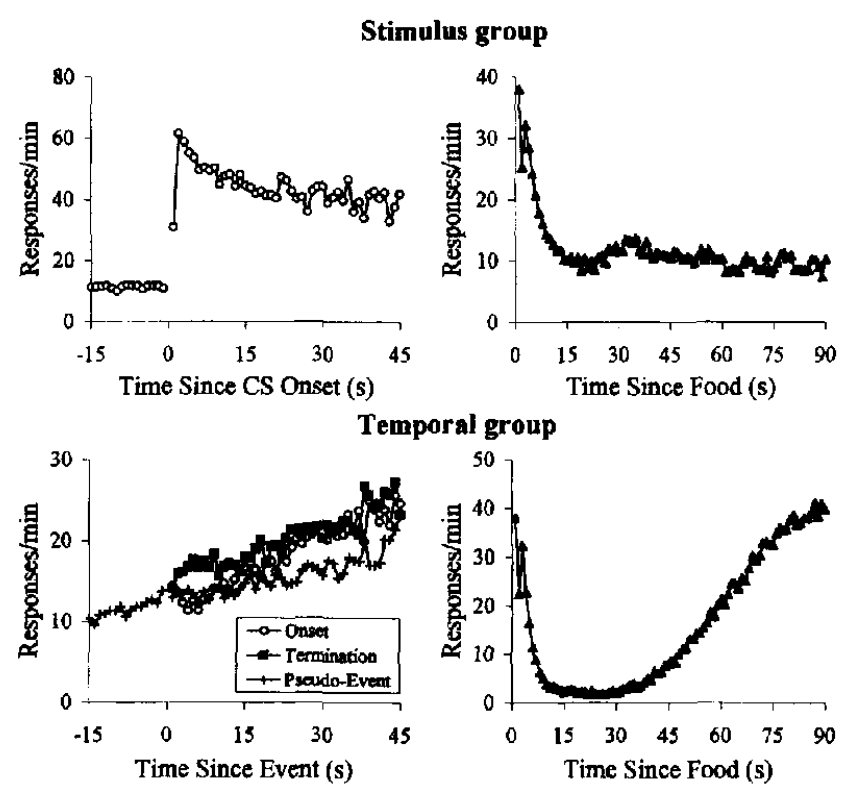

Figure 3. Top-left panel (stimulus group): local response rate in successive 1-s intervals, relative to the time of conditioned stimulus (CS) onset as a measure of control by the stimulus cue in Experiment 1. Top-right panel (stimulus group): local response rate in successive 1-s intervals, relative to the time of the previous food delivery as a measure of control by the random food-food interval. Bottom-left panel (temporal group): local response rate in successive 1-s intervals, relative to the time of CS onset, CS termination, or a pseudoevent as a measure of control by the irrelevant CS onset and CS termination events. Bottom-right panel (temporal group): local response rate in successive 1-s intervals, relative to the time of the previous food delivery as a measure of control by the temporal cue. 
presence of the CS. There was an initial bout of food cup responding, probably because of consumption of the previously delivered food, followed by a relatively constant rate of responding at around 10 responses $/ \mathrm{min}$.

Control by the temporal cue in the temporal group (bottom-right panel, Figure 3) was apparent because of the gradual increase in response rate from 30 to $90 \mathrm{~s}$, which occurred after an initial bout of food cup responding in the first $10 \mathrm{~s}$. Control by the irrelevant CS-onset and CStermination events in the temporal group is plotted in the bottom-left panel of Figure 3. The response rate functions for a pseudoevent are included as a baseline estimate of responding that was not controlled by the CS events (see Data Analysis section). The baseline rates of responding gradually increased as a function of time, because of the timing of the food-food interval (the temporal cue). After CS onset, response rates were similar to baseline for the first $15 \mathrm{~s}$ and then increased to a level that was about four responses/min above baseline for the remainder of the interval. There was an immediate increase in response rate following CS termination that was fairly constant at around four responses/min.

These descriptive aspects of the data were verified by an analysis of variance (ANOVA) that was conducted on the response rates following CS onset, CS termination, or the pseudoevent, averaged over 15-s blocks of time (1-15 s, 16-30 s, and 31-45 s). The analysis revealed significant main effects of event type, $F(2,14)=23.9, p<.001$, and time since event, $F(2,14)=29.0, p<.001$, and a significant interaction, $F(4,28)=4.5, p<.01$. Tukey post hoc analyses contrasting the three events at each time block revealed that during Seconds 1-15, response rates following CS termination were significantly greater than response rates following both CS onset and the pseudoevent, which were not different from one another. During 16-30 s and 31-45 s, response rates following both CS onset and CS termination were significantly greater than response rates following the pseudoevent, but CS onset and termination did not differ from each other.

Compound training. The stimulus and temporal DRs over the course of compound training (Blocks 31-60) are plotted in Figure 2. The stimulus and temporal DRs over Blocks 51-60 were tested against a chance level of .5. All of the DRs exceeded chance levels in all groups, all $t s(7)>4.8$, all $p s<.001$, except for the stimulus DR produced by the temporal group (middle panel). These rats failed to respond above chance to the stimulus cue in the compound, $t(7)=1.9$.

An ANOVA was conducted on the mean stimulus and temporal DRs over Blocks 51-60 of compound training with a between-subjects variable of group and a repeated measures variable of cue type. There was a significant main effect of cue type, $F(1,21)=25.9, p<.001$, and a significant Cue Type $\times$ Group interaction, $F(2,21)=7.6$, $p<.01$. Tukey post hoc analyses indicated that the Cue Type $\times$ Group interaction was due to significantly lower stimulus DRs in the temporal group compared with the stimulus and control groups $(p<.01)$, but there were no differences in the temporal DRs among the three groups. Within-group $t$ tests were also conducted to compare the stimulus and temporal DRs. In all three groups of rats, the temporal DR was significantly higher than the stimulus $\mathrm{DR}$, all $t \mathrm{~s}(7)>$ 2.5 , all $p \mathrm{~s}>.05$.

\section{Discussion}

Pretraining with a stimulus conditioning procedure resulted in robust conditioning and a temporal gradient with a shape that was consistent with control by the CS. Specifically, response rates rose abruptly within 1 to $2 \mathrm{~s}$ after CS onset, declined slightly until approximately $15 \mathrm{~s}$ after CS onset, and then remained relatively constant over the remainder of the CS. Pretraining with a temporal conditioning procedure resulted in robust conditioning and a temporal gradient with a shape that was consistent with control by time since the previous food, with a reactive component to the previous reinforcer and an anticipatory response to the upcoming reinforcer. There was an initial bout of food cup checking followed by a period of near-zero responding; then response rates increased gradually from 30 to $90 \mathrm{~s}$.

Pretraining with the temporal conditioning procedure resulted in poorer control by the stimulus cue, compared with groups that received either the stimulus cue or no cues in the pretraining phase. However, pretraining with the stimulus cue did not interfere with temporal conditioning. The results are consistent with the report by Williams and LoLordo (1995) that temporal cues interfered with the acquisition of responding to a CS, but not vice versa.

There are two primary interpretations of the interference in stimulus conditioning in the temporal group: blocking and learned irrelevance. With regard to blocking, there are two likely mechanisms: (a) The learning of the temporal cue that occurred with the fixed food-food interval may have interfered with later stimulus conditioning. Williams and LoLordo (1995) contended that the interference in conditioning occurred because the temporal cue blocked conditioning to the CS. The implication is that a temporal interval could interfere with learning to respond to a CS, much like one CS might interfere with learning to respond to a second CS. (b) There may have been conditioning to the experimental context that later blocked acquisition to the stimulus. The delivery of a US at random times during a pretraining phase can produce a detriment in the speed of acquisition of CRs to a CS, when the CS is paired with the US in a later phase (the US preexposure effect; Kamin, 1961; Kimble \& Dufort, 1956; Pavlov, 1927; Randich \& Ross, 1985). One interpretation of the US preexposure effect is that the delivery of the US alone results in conditioning to the experimental context (Rescorla \& Wagner, 1972). The context then acts like a CS in the second phase, blocking acquisition to a novel CS.

One means of discriminating between the two blocking interpretations would be to determine whether blocking would occur if the temporal conditioning procedure involved the delivery of a variable food-food interval. The context blocking hypothesis would predict the same degree of blocking by exposure to a fixed and variable food-food interval, provided that the density of food delivery was the same. In contrast, if temporal conditioning to the fixed food-food interval blocked stimulus conditioning, then the 
interference would not occur or would be less extensive with a variable food-food interval.

An alternative interpretation of the interference in stimulus conditioning in the temporal group is that this group learned that the CS was irrelevant in pretraining. The temporal conditioning procedure consisted of the delivery of the CS at random times in the food-food interval, which can impair conditioning to the CS when it is paired with the US in a second phase (Bennett, Maldonado, \& Mackintosh, 1995; Bonardi \& Hall, 1996; Kremer, 1971; Mackintosh, 1973; Matzel, Schachtman, \& Miller, 1988). Learned irrelevance could explain why stimulus conditioning in the temporal group was impaired.

A second finding was that the temporal cue exerted stronger control over behavior in the compound, regardless of whether the rats received the temporal cue, stimulus cue, or no cues in the pretraining phase. The difference in salience of the stimulus and temporal cues may have been a factor in the observed asymmetry in cue competition (Kamin, 1968, 1969; Schreurs \& Gormezano, 1982). The temporal cue may have been more salient because it provided better information in predicting food delivery than the stimulus cue (e.g., Egger \& Miller, 1963). Figure 4 diagrams the information provided by the stimulus and temporal cues in the stimulus and temporal conditioning
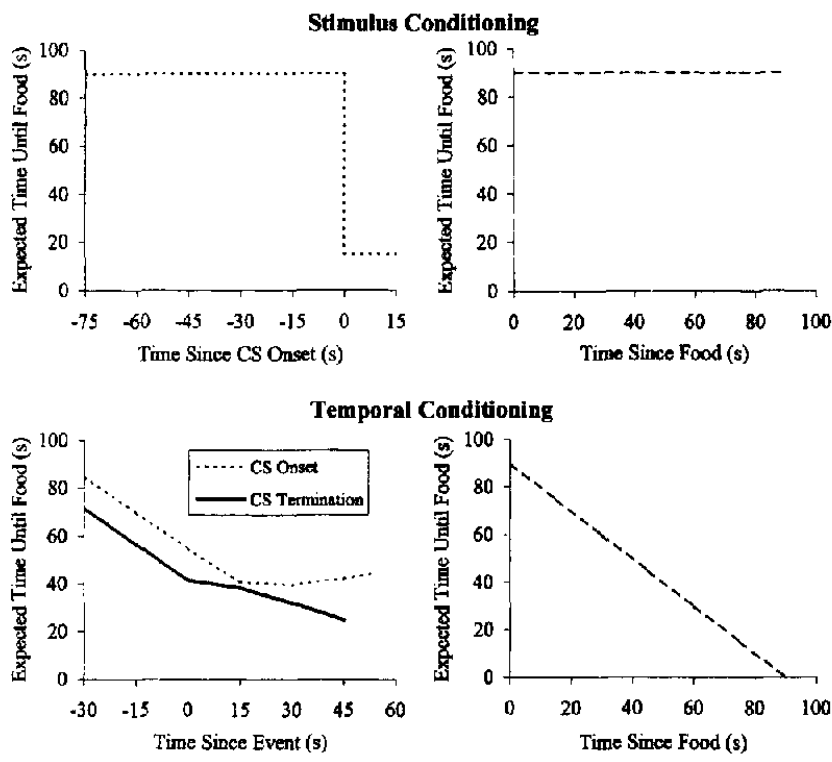

Figure 4. Top-left panel: the expected time until food as a function of time since conditioned stimulus (CS) onset for the stimulus conditioning procedure in Experiment 1; CS onset would occur at $0 \mathrm{~s}$. Top-right panel: the expected time until food as a function of time since the previous food delivery for the random $90-s$ food-food interval in the stimulus conditioning procedure. Bottom-left panel: the expected time until food as a function of time since either CS onset or termination in the temporal conditioning procedure; CS onset or termination would occur at $0 \mathrm{~s}$. Bottom-right panel: the expected time until food as a function of time since the previous food delivery for the fixed 90-s food-food interval in the temporal conditioning procedure. procedures, respectively. The top-left panel shows the expected time until food as a function of time since CS onset in the stimulus conditioning procedure. The expected time until food was $90 \mathrm{~s}$ in the absence of the CS and then dropped to $15 \mathrm{~s}$ at CS onset and remained there during the duration of the CS. The shape of the function is created by the random 90-s food-food and random 15-s CS onset-food intervals. The hazard function for the exponential distribution is constant, meaning that there is no change in information from moment to moment. With no local predictability of the time of food delivery, the best estimate of the time of food would be equal to the mean time between successive food deliveries-at all times.

The bottom-right panel of Figure 4 shows the expected time until food as a function of time since food delivery in the temporal conditioning procedure. The expected time until food decreased linearly as a function of time since food. The shape of the function was created by the fixed interval between successive food deliveries. If the rat were capable of timing the interval perfectly, the subjective time remaining until food would decrease veridically with the elapsed time in the interval. (The expected time functions for the irrelevant $\mathrm{CS}$ onset and termination events are discussed below.)

The expected time functions provide some insight as to why the temporal cue might be more salient than the stimulus cue. There are two key differences in the expected time functions for the stimulus and temporal cues. First, the temporal cue provides information about the upcoming availability of food farther in advance than the stimulus cue. The temporal cue provides information throughout the food-food interval, whereas the stimulus cue provides information only during the terminal portion. Second, the temporal cue provides a more accurate prediction of the time of food delivery. If the rats were capable of timing the fixed $90 \mathrm{~s}$ food-food interval perfectly, then the time of the next food delivery could be determined exactly. On the other hand, the stimulus cue allows only for a determination of the time of the upcoming food within $15 \mathrm{~s}$. Either of these differences in the predictability of food may have produced a preference for responding to the temporal cue in the compound regardless of any training history with the stimulus cue.

The expected time functions also provide an interpretation of the response rate functions. The shape of the response rate function in the stimulus group (Figure 3, top-left panel), which was interpreted as responding to the CS as a whole, could alternatively be interpreted as timing from CS onset. The shape of the response rate function (a square wave) is the same as the shape of the expected time function for the stimulus conditioning procedure. The expected time function for the temporal conditioning procedure could also explain the increasing response rate function produced by the temporal group during the food-food interval. Here, too, the shape of the response rate function produced by the rats is similar to the shape of the expected time function. If one were to assume that the rats were timing relative to CS onset (stimulus conditioning) and food delivery (temporal conditioning), and tracking the expected time to food associated 
with those events, then one would not need to invoke an additional association of the CS as a whole with food. A timing account provides a unified description of the results from the two procedures using the same mechanism.

Tracking of the expected time functions may also explain the shape of the response rate functions after the irrelevant events in each procedure. In the stimulus conditioning procedure, the expected time until food for the food-food interval remained constant at $90 \mathrm{~s}$ (top-right panel of Figure 4). The corresponding response rate function was constant over the food-food interval (top-right panel of Figure 3).

In the temporal conditioning procedure, if the noise had occurred at random times throughout the food-food interval with no restrictions on the time of onset or duration of the noise, then the expected time functions for the CS onset and termination events would be constant at $45 \mathrm{~s}$. However, there were two restrictions in the scheduled times of CS onset and termination. First, CS onset never occurred in the last $15 \mathrm{~s}$ of the food-food interval; this is because CS onset was determined by a uniform distribution ranging from 0 to $75 \mathrm{~s}$ from the prior food delivery. Therefore, the time from CS onset to food was fixed $15+$ Uniform $(0-75) \mathrm{s}$, which resulted in an expected time function that decreased from 54.7 to $40.7 \mathrm{~s}$ over the first $15 \mathrm{~s}$ and then remained relatively constant at around $40 \mathrm{~s}$. Second, if the sum of the pre-CS and CS durations exceeded $90 \mathrm{~s}$, then a new sample was drawn; this resulted in fewer CS onset times late in the food-food interval than would be expected in the absence of restrictions. As a result of the restrictions, the expected time function for CS onset increased slightly during the last $20 \mathrm{~s}$ of the CS onset-food interval. The expected time function for CS termination fell gradually from 41.5 to 24.9 s over the CS termination-food interval. Because CS termination could only occur once a CS onset had occurred, the distribution of times between CS termination and food is a convolution of a Uniform 0-75 $\mathrm{s}$ (with sampling bias) and a random 15-s interval. This distribution increases slightly and then falls gradually, which creates an expected time function that falls gradually.

Response rates relative to CS onset and CS termination were greater than response rates relative to the pseudoevent (see Figure 3, bottom-left panel), indicating that the rats may have been using the irrelevant cues (and tracking their expected time functions) as an additional source of information in predicting the time of the upcoming reinforcer. However, the expected time functions are too complicated to determine how timing from the irrelevant CS events combines with the timing of the food-food interval.

\section{Experiment 2}

Two major changes in experimental design were introduced in Experiment 2 to determine the source of (a) the interference of responding to the stimulus cue when rats were trained with the temporal conditioning procedure, and (b) the dominance of the temporal cue regardless of training history.

The interference in conditioning to the stimulus cue may have been due to blocking by the temporal cue or learned irrelevance of the stimulus cue in pretraining. Accordingly, another control group was added to assess the role of learned irrelevance of the noise in the temporal conditioning procedure. The learned irrelevance (LI) control group received the temporal cue in pretraining, but did not receive any CS presentations. If learned irrelevance of the CS occurs in the temporal conditioning procedure, then the LI control group should exhibit better performance to the CS in the compound than the temporal group.

The source of the dominance of the temporal cue was assessed by adding variability to the food-food interval in the temporal conditioning procedure in order to make the predictability of the temporal cue more equivalent to the stimulus cue. A fixed 75 + random 15 -s interval separated successive food presentations, resulting in an expected time function that decreased linearly until $15 \mathrm{~s}$ and then remained constant at $15 \mathrm{~s}$ until food was delivered. During the $15 \mathrm{~s}$ prior to food delivery, with perfect timing, the temporal cue is as precise a predictor of the upcoming food as the stimulus cue. If the difference in salience of the two cues was due to the accuracy of the prediction of food delivery, then the addition of variability should result in weak control by the temporal cue, thereby allowing for acquisition to the stimulus cue in the compound.

The added variability does not change the density of food delivery, which presumably affects the strength of context conditioning (Goddard \& Jenkins, 1988; Tomie, 1981); it also does not alter the degree to which the temporal cue predicts food delivery in advance of the onset of the stimulus. If either of these factors is important in determining the salience of the temporal cue, then we would expect to replicate the results of Experiment 1, even with the addition of variability to the food-food interval.

In addition to the above changes, the delivery of the irrelevant noise in the temporal conditioning procedure was altered to correct for the sampling bias. There were no restrictions on the CS onset or termination time within the food-food interval, nor was there any restriction of the number of $\mathrm{CS}$ occurrences during the food-food interval. As a result of this change, the mean time between CS onset (or CS termination) and upcoming food delivery was $46.25 \mathrm{~s}$, and the expected time from both CS onset and CS termination was constant at $46.25 \mathrm{~s}$, because both events occurred at random with respect to the time of food delivery. ${ }^{3}$ The

\footnotetext{
${ }^{3}$ In the temporal conditioning procedure, the expected time between successive reinforcers was fixed $75+$ random $15 \mathrm{~s}$. At a random time during the session, the probability of receiving a $\mathrm{CS}$ onset (or termination) during the fixed 75-s portion of the food-food interval is $5 / 6$ and the probability of receiving a CS onset (or termination) during the random $15 \mathrm{~s}$ portion is $1 / 6$. If CS onset (or termination) were to occur in the fixed 75-s portion of the food-food interval, then the mean time until the next food delivery would be $37.5 \mathrm{~s}+15 \mathrm{~s}=56.5 \mathrm{~s}$ (the $37.5 \mathrm{~s}$ is the mean of the uniformly distributed times from $0-75 \mathrm{~s}$, and the $15 \mathrm{~s}$ is the mean of the random 15-s portion of the food-food interval). If CS onset were to occur in the random 15-s portion of the food-food interval, then the mean time until the next food delivery would be $15 \mathrm{~s}$. The overall mean of the distribution would therefore be $(5 / 6 \times 56.5 \mathrm{~s})+$ $(1 / 6 \times 15 \mathrm{~s})=46.25 \mathrm{~s}$.
} 
change in the scheduled delivery of the CS should remove any local predictability of the time of CS onset or termination, the duration of the CS, and the time of food delivery. Moreover, by making the expected time functions identical for the two events, the response rate functions relative to these events may be more similar.

\section{Method}

\section{Subjects}

The experimental animals were 32 male Sprague-Dawley rats (Taconic Laboratories, Germantown, NY), age 64 days at the beginning of the experiment. All aspects of their care and maintenance were the same as in Experiment 1.

\section{Apparatus}

The 12 operant chambers that were used in Experiment 1 served as the experimental apparatus. Delivery of stimuli and reinforcers and recording of responses was conducted in the same fashion as in Experiment 1.

\section{Procedure}

The rats were randomly assigned to a stimulus group, a temporal group, an LI control group, or an untreated control group. The stimulus conditioning procedure was identical to Experiment 1. The other procedures were somewhat different (see Figure 5). First, the food-food interval in the temporal and LI control groups and in the compound conditioning procedure was changed from fixed $90 \mathrm{~s}$ to fixed $75+$ random $15 \mathrm{~s}$. Second, the delivery of the CS in the temporal conditioning procedure was altered slightly: The random 15-s noise was delivered independently of food, and there was no

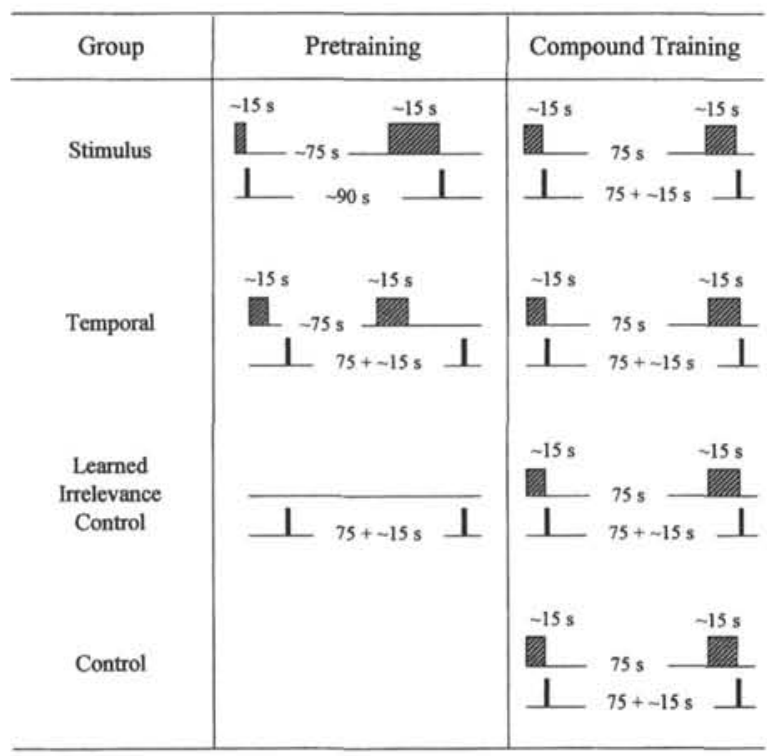

Figure 5. The schedules for delivery of the conditioned and unconditioned stimulus to the stimulus group, temporal group, learned irrelevance control group, and (untreated) control group during the pretraining and compound training phases of Experiment 2 . restriction as to how many times the noise could occur in the food-food interval. The interval between the termination of the previous noise and the onset of the next noise was random $75 \mathrm{~s}$.

The rats in the stimulus, temporal, and LI control groups received their respective pretraining procedures for six daily sessions, whereas the rats in the control group were placed in the boxes without receiving any stimuli or reinforcements. During compound training, all four groups of rats received the compound conditioning procedure for six sessions. Approximately 79 food-food cycles were delivered in each 2-hr session. All other features of Experiment 2 were conducted in the same manner as Experiment 1 .

\section{Results}

\section{Pretraining}

Discrimination ratios. The stimulus and temporal DRs (see Figure 6) were calculated as in Experiment 1. By the end of pretraining (Blocks 21-30), the stimulus DR was significantly greater than the temporal DR in the stimulus group, $t(7)=4.9, p<.01$; the temporal DR was significantly greater than the stimulus DR in the temporal group, $t(7)=4.3, p<.01$; and the temporal DR in the LI control group was similar to the temporal DR in the temporal group, $t(14)=0.8$.

The DRs over Blocks $21-30$ of pretraining were tested against the chance level of .5. All of the DRs in all groups were above chance, all $t \mathrm{~s}(7)>5.2$, all $p s<.001$, except the temporal DR in the stimulus group, $t(7)=1.2$. The above-chance stimulus DR in the temporal group indicates that there was a higher response rate during the irrelevant CS compared with the background, a result that will be examined more closely in the local response rate functions below.

Local response rate functions. The local response rate functions for control by CS onset, CS termination, and food delivery are displayed in Figure 7. In the stimulus group (top-left panel), CS onset produced a sharp increase in response rate from approximately 5 responses/min to approximately 50 responses/min, followed by a decline over the next $10-15 \mathrm{~s}$ of the CS, and then a relatively constant rate of around 30 responses/min thereafter.

Control by the temporal cue was evident in the temporal group (bottom-right panel) and in the LI control group- the two functions were similar. The mean response rates plotted as a function of time since the previous food delivery were initially high, followed by a near-zero rate of responding from 10 to $30 \mathrm{~s}$, and then followed by an increasing response rate. The response rate functions reached a maximum of around 20 responses $/ \mathrm{min}$ at $75 \mathrm{~s}$ after the prior food delivery and then remained relatively constant. Both of the gradients in Experiment 2 were flatter and lower than the gradient produced by the temporal group in Experiment 1 during the last $15 \mathrm{~s}$ of the food-food interval.

The response rate function for control by the random 90-s food-food interval in the stimulus group is shown in the top-right panel of Figure 7. There was an initial high rate of food-cup behavior, probably caused by the rat eating the food pellet, followed by a relatively constant, nonzero rate of responding, which was maintained over the duration of the food-food interval. 
The response rate functions produced by the temporal group relative to the time of CS onset, CS termination, or a pseudoevent (see the Data Analysis section in Experiment 1) are displayed in the bottom-left panel of Figure 7. The baseline rates of responding gradually increased because of
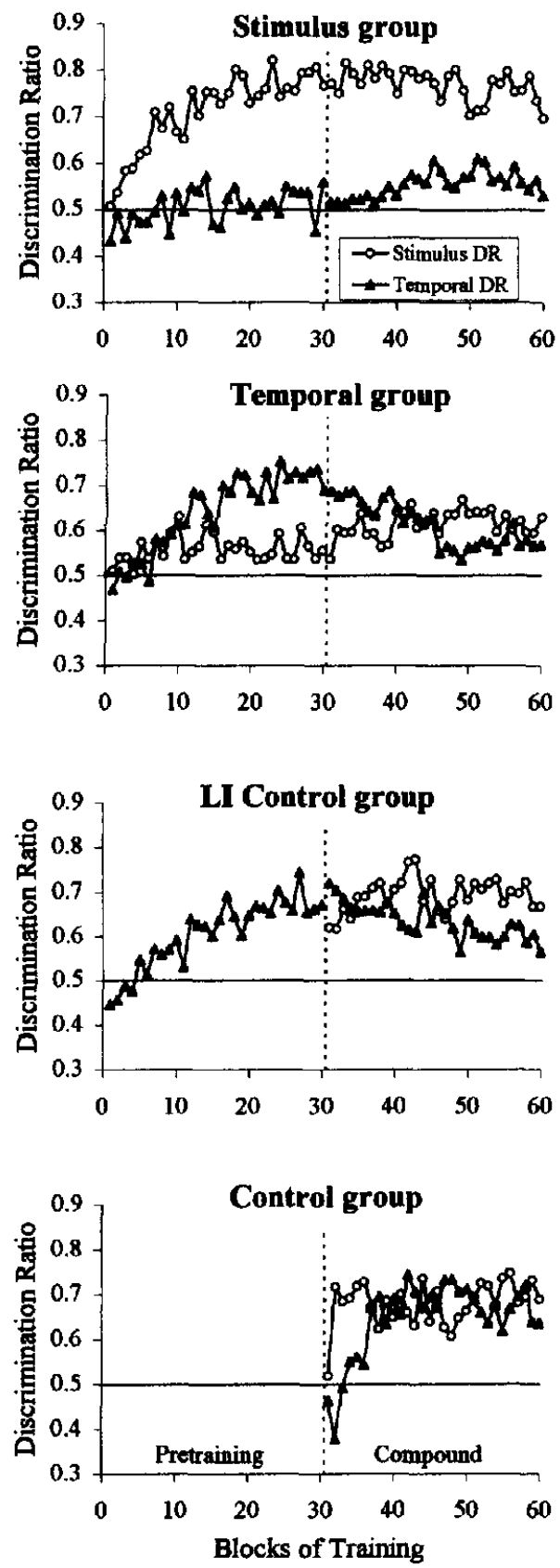

Figure 6. Acquisition of stimulus or temporal discrimination ratios (DRs) during pretraining (Blocks 1-30) and compound training (Blocks 31-60) in Experiment 2. Each panel displays the DRs for one of the four groups of rats. The strength of the stimulus DR is a measure of the degree of control by the stimulus cue and the strength of the temporal DR is a measure of the degree of control by the temporal cue. The chance level of 0.5 is indicated by the solid line.
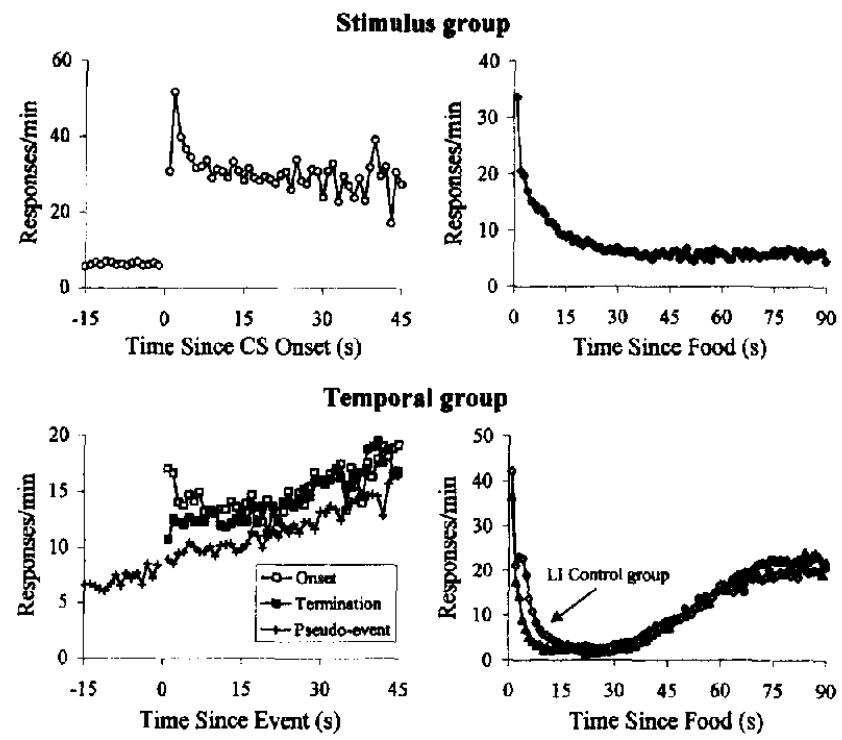

Figure 7. Top-left panel (stimulus group): local response rate in successive $1-s$ intervals, relative to the time of conditioned stimulus (CS) onset as a measure of control by the stimulus cue in Experiment 2. Top-right panel (stimulus group): local response rate in successive 1-s intervals, relative to the time of the previous food delivery as a measure of control by the random food-food interval. Bottom-left panel (temporal group): local response rate in successive 1-s intervals, relative to the time of CS onset, CS termination, or a pseudoevent as a measure of control by the irrelevant CS onset and $\mathrm{CS}$ termination events. Bottom-right panel (temporal group): local response rate in successive $1-\mathrm{s}$ intervals, relative to the time of the previous food delivery as a measure of control by the temporal cue.

timing of the food-food interval. Both CS-onset and CStermination events produced a small (four responses/min) increase in response rate that was maintained relative to the baseline rate.

An ANOVA was conducted on the response rate functions after CS onset, CS termination, or the pseudoevent, with within-subjects variables of event type and time since event (1-15 s, 16-30 s, and 31-45 s). The analysis revealed significant effects of event type, $F(2,14)=20.1, p<.001$; time since event, $F(2,14)=19.6, p<.001$; and Event Type $\times$ Time Since Event, $F(4,28)=4.1, p<.05$. Tukey post hoc analyses compared response rates following the three events at each block of time. During all three time blocks, response rates after both CS onset and termination were greater than response rate following the pseudoevent. During Seconds $1-15$, response rates following CS onset were greater than response rates following CS termination, reflecting the higher response rate during the first $5 \mathrm{~s}$ after CS onset.

\section{Compound Training}

The stimulus and temporal DRs during compound training (Blocks 31-60) are displayed in Figure 6. The mean stimulus and temporal DRs over Blocks 51-60 were tested against the chance level of .5. All of the DRs were above chance in all four groups, all $t s>2.8$, all $p s<.05$. 
A two-way ANOVA with the factors of group and cue type was conducted on the stimulus and temporal DRs over Blocks 51-60 of compound training. There was a significant main effect of cue type, $F(1,28)=22.2, p<.001$, which was due to greater control by the stimulus cue $(M=0.69)$ than the temporal cue $(M=0.60)$. However, neither the group main effect, $F(3,28)=2.6$, nor the Cue Type $\times$ Group interaction, $F(3,28)=2.6$, were significant. Two additional $t$ tests were conducted to address the key issues of the experiment: The stimulus DRs of the LI control group were not statistically different from the stimulus DRs of the temporal group, $t(14)=1.5$, and there was a significant decrement in the temporal DRs in the stimulus group compared with the control group, $t(14)=2.6, p<.05$.

\section{Discussion}

The results of Experiment 2 provided additional support for tracking of the expected time to food relative to multiple events. The change in the food-food interval to fixed 75 random $15 \mathrm{~s}$ in Experiment 2 flattened the expected time function during the last $15 \mathrm{~s}$ (Figure 4, bottom-right panel). The same shape was observed in the response rate function, which increased from 30 to $75 \mathrm{~s}$ and then was flat during the last $15 \mathrm{~s}$.

The rats also responded to the expected time to food for the irrelevant events. The rats in the stimulus group responded at a constant low rate in the random 90 -s food-food interval, which had an expected time function that was constant at $90 \mathrm{~s}$ (Figure 4, top-right panel). The expected time analysis provides an explanation of both the shape and rate of responding. The shape would be flat because of the constant expected time to food at all times; the rate would be low because of the relatively long expected time until food. An even more compelling result was responding by the temporal group to the irrelevant CS-onset and CStermination events. There was a small, constant increase in responding after both events, relative to baseline rates. The expected time function for these two events was constant at $46.25 \mathrm{~s}$. The fact that there was a constant increase in responding, relative to both $\mathrm{CS}$ events, indicates that there may be some sort of additive response rule when two events are being timed.

One goal of Experiment 2 was to reduce the salience of the temporal cue by adding variability to the food-food interval. The addition of variability to the food-food interval resulted in stronger control by the stimulus and an interference in conditioning to the temporal cue in the stimulus group, which may have been due to blocking by the stimulus cue.

The pattern of results suggests that there was a reversal in the relative salience of the two cues, due to the addition of variability in the temporal cue. The results are reminiscent of blocking in simultaneous compounds when there are differences in the salience of two CSs: There are often asymmetries in blocking so that the more salient CS produces blocking of the less salient CS but not vice versa (e.g., Kamin, 1968, 1969; Schreurs \& Gormezano, 1982). The results of Experiment 2 indicate that the predictive accuracy of the upcoming food delivery (see Figure 4) is at least one factor in determining the salience of the temporal cue. The results rule out context conditioning as a mechanism for producing the dominance of the temporal cue.

Another goal of Experiment 2 was to determine whether the interference in stimulus conditioning in the compound was due to blocking by the temporal cue or learned irrelevance of the CS. There were no differences in performance to the stimulus cue during compound training in the temporal and LI control groups, indicating that learned irrelevance was not a substantial contributor in Experiment 2. Given that the delivery of the irrelevant CS removed any local predictability by eliminating the sampling restrictions, one would expect that learned irrelevance would have been more likely in Experiment 2. However, the results do not directly reflect any degree of learned irrelevance that may have occurred in the temporal group in Experiment 1.

\section{General Discussion}

\section{Control by Time Since an Event}

Five findings in the two experiments were consistent with tracking of the expected time functions: (a) In the stimulus conditioning procedure, the expected time until food abruptly decreased at stimulus onset and then remained constant at 15 s (top-left panel of Figure 4); response rates in the stimulus group in both experiments increased abruptly after CS onset and then remained relatively constant, albeit some modest decline during the first 10-15 s (top-left panel of Figures 3 and 7). (b) In the temporal conditioning procedure in Experiment 1, the expected time until food gradually decreased as a function of time since food delivery (bottomright panel of Figure 4); response rates in the temporal group gradually increased as a function of time since food delivery (bottom-right panel of Figure 3). (c) In the temporal conditioning procedure in Experiment 2, the expected time until food gradually decreased over the first $75 \mathrm{~s}$ and then remained constant at $15 \mathrm{~s}$; response rates gradually increased over the first $75 \mathrm{~s}$ of the food-food interval, and then remained relatively constant over the last $15 \mathrm{~s}$ (bottom-right panel of Figure 7). (d) The expected time function for the random food-food interval in the stimulus conditioning procedure was constant at $90 \mathrm{~s}$ (top-right panel of Figure 4); response rates were relatively constant when plotted relative to the time of the prior food delivery (top-right panel of Figures 3 and 7). (e) The expected time function for the irrelevant CS-onset or CS-termination events in the temporal conditioning procedure was constant at $46.25 \mathrm{~s}$ in Experiment 2; there was a small constant increase in responding as a function of time since CS onset or termination (bottom-left panel of Figure 7).

Conventional accounts of these varied results would rely on varied mechanisms. For example, in the stimulus conditioning procedure, response rate abruptly increases after CS onset because food is associated with the CS; in the temporal conditioning procedure, response rate gradually increases because food is associated with the time since the prior food delivery; a relatively constant response rate during a random 
food-food interval is due to either contextual conditioning to the experimental apparatus or to baseline rates of responding that are not under experimental control; the increase in responding following the irrelevant CS-onset event is due to the unconditioned excitatory effect of a stimulus, such as stimulus intensity dynamism.

The expected time analysis provides a unified account that does not rely on special rules to explain responding relative to different events or to different distributions of intervals. It predicts that the response rate at any moment in time is related to the expected time until the next food delivery, relative to the time of occurrence of a previous event (CS onset, CS termination, or food delivery). Tracking of the distribution of interreinforcement intervals has been reported in operant schedules of reinforcement (e.g., Catania \& Reynolds, 1968; Lund, 1976) as well as in classical conditioning (LaBarbera \& Church, 1974; Libby \& Church, 1975), indicating that expected time tracking may be a ubiquitous property of the timing mechanism in both classical and operant procedures. The expected time function would be affected by the duration, variability, and distribution form of the scheduled intervals, properties that are related to the shape of the response rate function (Brunner, Fairhurst, Stolovitzky, \& Gibbon, 1997; Church, Lacourse, \& Crystal, 1998).

The question then becomes one of credit assignment: What factors will determine whether an animal will respond to the food-delivery, CS-onset, or CS-termination events? There are a number of possible answers. (a) Perhaps the event that is most contiguous with US delivery gains control over behavior. This does not seem plausible because the temporal cue exerted greater control over behavior than the more contiguous stimulus cue (regardless of prior training history) in the compound training phase of Experiment 1. Moreover, in the temporal conditioning procedure in both experiments, the irrelevant stimulus cue was actually more contiguous with reinforcement (on average) than the prior food-delivery event, yet the food-delivery event exerted substantial control over behavior. (b) Perhaps, instead, only events that occur at a fixed duration prior to food delivery gain control over behavior. Normally, it is assumed that temporal conditioning occurs only when there is a fixed interval between successive USs. However, in Experiment 2, temporal conditioning was observed in a partially random food-food interval, and, it is possible that temporal conditioning occurred in the random 90-s food-food interval as well. (c) The most plausible hypothesis is that all events gain control over behavior, with the strength and form of CRs shaped by a combination of the expected time functions for multiple intervals. There were a number of demonstrations of control by multiple events in the same animal in the present experiments. For example, there appeared to be control by the CS event(s) and food-delivery event in both pretraining procedures (see Figures 3 and 7). Moreover, the rats in the control group exhibited control by both the stimulus and temporal cues in the compound training phase in both experiments, and, the group that was trained with the weaker cue (the stimulus group in Experiment 1 and the temporal group in Experiment 2) demonstrated substantial learning of the previously irrelevant cue during compound training.

If the rats were tracking the expected time functions, relative to CS-onset, CS-termination, and food-delivery events, then they must have been timing the duration of multiple intervals: CS onset-food, CS termination-food and food-food (they may have also been timing CS onset-CS termination, food-CS onset and food-CS termination). Rats can simultaneously time at least three different intervals between events and upcoming reinforcers in both instrumental (Leak \& Gibbon, 1995; Meck \& Church, 1984) and classical (Desmond \& Moore, 1991; Kehoe et al., 1989; Millenson, Kehoe, \& Gormezano, 1977) procedures. Moreover, recent work by Miller and colleagues (cf. Savastano \& Miller, 1998) has shown that temporal relations are learned between events that occur closely in time, allowing for prediction of the time of occurrence of the US. These results are all indicative of simultaneous timing of multiple intervals between CS events and US delivery. The present results expand on the earlier findings by demonstrating timing of multiple intervals, even in cases in which there is a presumed random or uncorrelated relationship between the event and upcoming food delivery.

Simultaneous temporal processing may be an important contributor to the conditioning process in many conditioning paradigms. If rats time the CS onset-food, CS terminationfood, and food-food intervals, then there should be a straightforward combination rule for determining the relative influence of the various intervals on responding. The responding of the temporal group to the irrelevant CS events in pretraining of Experiment 2 (bottom-left panel of Figure 7) suggests that some sort of additive combination rule may apply when two or more intervals are being simultaneously timed. However, there may be factors such as variability or prior training history that affect the weight given to each interval. The cue interaction effects provide one means of assessing the relative contribution of the CS onset-food and food-food intervals.

\section{Cue Interaction Effects}

In Experiment 1, pretraining with the temporal conditioning procedure interfered with control by the stimulus cue during the compound training phase. Although this interference may have been due to blocking by the temporal cue, learned irrelevance of the stimulus cue, or a performance failure (Savastano \& Miller, 1998), the more remote food delivery event clearly controlled greater responding than the more contiguous stimulus event.

In related research on serial compound conditioning, Kehoe, Schreurs, and Amodei (1981, Experiment 1) trained rabbits in an initial phase with an $800-\mathrm{ms} \mathrm{CS}_{1}$ that was followed immediately by a shock US; the conditioned response was retraction of the nictitating membrane. In a subsequent compound training phase, a $\mathrm{CS}_{2}$ was delivered in an overlapping serial compound arrangement in which the $\mathrm{CS}_{2}$ was turned on during the last $400 \mathrm{~ms}$ of the $\mathrm{CS}_{1}$. Thus, $\mathrm{CS}_{2}$ onset was more contiguous with the occurrence of the shock US, but both $\mathrm{CS}_{1}$ and $\mathrm{CS}_{2}$ terminated at the time of US 
occurrence. There was no evidence of acquisition to $\mathrm{CS}_{2}$ in the overlapping compound.

Our compound training procedure is similar to an overlapping serial compound (as are many conditioning procedures) because the stimulus cue occurred at the end of the food-food interval. The comparability of the overlapping serial compound research and our compound conditioning results suggest that the particular events (US, $\mathrm{CS}_{1}, \mathrm{CS}_{2}$ ) that mark the intervals may be less important than the intervals themselves.

The effects of variability in the food-food interval on the direction of cue competition indicate that temporal predictability is an important factor in determining the salience of the temporal cue. A timing analysis that incorporates the temporal information provided by the stimulus and temporal cues can predict the direction of cue competition. There are two sources of variability that could affect the salience of the temporal cue: (a) experimentally induced variability in the scheduled intervals, and (b) internal variability in the perception of time. Animals (and people) are incapable of timing intervals with complete precision. The error in timing increases proportionately with the mean interval duration (the scalar property; Gibbon, 1977).

By considering both sources of variability, one can predict the direction of competition between the stimulus and temporal cues. In Experiment 1, there was no programmed variability in the food-food interval, therefore the only source of error in timing would be due to internal variability in the perception of the 90-s interval. On the other hand, there were two sources of variability in timing of the CS onset-food interval-internal variability in the timing system and variability in the scheduled intervals. The experimentally induced variability would be equal to $15 \mathrm{~s}$ because the standard deviation of a random interval is equal to its mean (Evans, Hastings, \& Peacock, 1993). Thus, the dominance of the temporal cue in Experiment 1 may have occurred because the temporal cue was a more precise predictor of the time of food delivery than the stimulus cue, perhaps because there was only one source of variability in the food-food interval.

In Experiment 2, the temporal cue and stimulus cue had the same experimentally programmed variability, equal to $15 \mathrm{~s}$ (because of the random 15-s component of the temporal cue and random 15-s CS duration). So, any difference in predictability of food must have arisen from internal variability in the perception of the scheduled intervals. Because the temporal cue was 6 times the duration of the stimulus cue, scalar variance would predict that the standard deviation of the perceived food-food interval would be 6 times the standard deviation of the perceived CS onset-food interval. Thus, the dominance of the stimulus cue in Experiment 2 may have occurred because the stimulus cue was a more precise predictor of the time of the upcoming food delivery, because of the lower degree of internal variability in the perception of the duration of the CS.

Although the expected time analysis of the cue competition effects is only speculative, it does allow for prediction of the effects of variability in the food-food interval on the direction of cue competition in the compound. Further experimental tests may facilitate the development of a precise quantitative account of the effects of variability on cue competition.

\section{Theoretical Relevance}

The present results indicate the need to account for three attributes of timing in delay conditioning procedures: (a) tracking of the expected time functions, (b) timing of multiple intervals with some (to-be-determined) combination rule, and (c) the effect of variability in the food-food interval on the direction and extent of cue competition during later training with a compound.

There are many computational models that contain both timing and conditioning features. Some of these contain only a single integrated mechanism (Blazis et al., 1986; Church \& Broadbent, 1990; Grossberg \& Schmajuk, 1989; Machado, 1997; Sutton \& Barto, 1981, 1990), whereas others contain two mechanisms (Desmond \& Moore, 1988; Gallistel \& Gibbon, 2000; Gibbon \& Balsam, 1981). (See Church \& Kirkpatrick, in press; Kirkpatrick \& Church, 1998, for a review and discussion.) Although each model has its particular strengths and weaknesses, as a class they are able to account for a wider range of phenomena than a model of conditioning or a model of timing alone. Many of these models can explain the phenomena of acquisition, extinction, partial reinforcement, and so forth; models that contain a competitive learning rule can explain phenomena such as blocking and overshadowing; many of the models can accommodate at least some of the effects of temporal variables on conditioning, and all of the models can produce temporal gradients of responding.

In their present form, none of these models could account for the results of the present studies. However, Moore and Choi (1997) presented a variant of the temporal difference real-time conditioning model (Sutton \& Barto, 1990) that produces simultaneous temporal processing of two intervals. Their model was developed to explain the fact that conditioned eyeblink responses are unimodal on training trials in a trace conditioning procedure, but are bimodal when longer duration probes are used (Desmond \& Moore, 1991). The bimodality in response times on probes is due to timing relative to both $C S$ onset and CS termination. The model accounted for this simultaneous temporal processing by using two temporal cascades-one initiated by CS onset and one initiated by CS termination. Each cascade resulted in a gradient of associative strengths, which were summed to produce the output. It is possible that the Moore and Choi model could be extended to account for the simultaneous timing relative to CS onset, CS termination, and food. The model is particularly interesting because it uses a summation process for combining timing of two events, much like the additional control that was observed in the timing of the irrelevant CS events (Figures 3 and 7). However, the model does not have a mechanism for tracking the expected time to food, nor does it have a mechanism that would produce the effects of variability on the direction of cue competition. Any conditioning or timing model will need to be greatly altered in order to accommodate the present results, as well 
as a wide range of phenomena related to the conditioning and timing of responses.

\section{Conclusions}

After describing the phenomenon of temporal conditioning with a fixed US-US interval, Pavlov (1927) concluded, "When we come to seek an interpretation of these results, it seems pretty evident that the duration of time has acquired the properties of a conditioned stimulus" (p. 41). Yet, the empirical evidence of the importance of temporal factors in acquisition of response strength and in the timing of responses has not been adequately incorporated in theories of classical conditioning. Theories that lack a representation of time cannot account for many of the present results, including tracking of expected times and the effect of variability in the food-food interval on the direction of cue competition in the compound. Theories that contain both an associative mechanism and a timing mechanism may be able to account for the results. Because the present results can be interpreted with a timing analysis that relies on tracking of expected times from multiple events, it is possible that a theory that contains only a timing mechanism could prove sufficient.

\section{References}

Bennett, C. H., Maldonado, A., \& Mackintosh, N. J. (1995). Learned irrelevance is not the sum of exposure to CS and US. Quarterly Journal of Experimental Psychology: Comparative and Physiological Psychology, 48B, 117-128.

Blazis, D. E. J., Desmond, J. E., Moore, J. W., \& Berthier, N. E. (1986). Simulation of the classically conditioned nictitating response by a neuron-like adaptive element: A real-time invariant of the Sutton-Barto model. Proceedings of the Eighth Annual Conference of the Cognitive Science Society. Hillsdale, NJ: Erlbaum.

Bonardi, C., \& Hall, G. (1996). Learned irrelevance: No more than the sum of CS and US preexposure effects? Journal of Experimental Psychology: Animal Behavior Processes, 22, 183-191.

Bouton, M. E., \& Nelson, J. B. (1998). Mechanisms of featurepositive and feature-negative discrimination learning in an appetitive conditioning paradigm. In N. A. Schmajuk \& P. C. Holland (Eds.), Occasion setting: Associative learning and cognition in animals (pp. 69-112). Washington, DC: American Psychological Association.

Brown, B. L., Hemmes, N. S., deVaca, S. C., \& Pagano, C. (1993). Sign and goal tracking during delay and trace autoshaping in pigeons. Animal Learning \& Behavior, 21, 360-368.

Brunner, D., Fairhurst, S., Stolovitzky, G., \& Gibbon, J. (1997). Mnemonics for variability: Remembering food delay. Journal of Experimental Psychology: Animal Behavior Processes, 23, 68-82.

Catania, C. A., \& Reynolds, G. S. (1968). A quantitative analysis of the responding maintained by interval schedules of reinforcement. Journal of the Experimental Analysis of Behavior, 11, 327-383.

Church, R. M., \& Broadbent, H. A. (1990). Alternative representations of time, number, and rate. Cognition, 37, 55-81.

Church, R. M., \& Kirkpatrick, K. (in press). Theories of conditioning and timing. In R. R. Mowrer \& S. B. Klein (Eds.), Contemporary learning: Theory and application. Hillsdale, NJ: Erlbaum.
Church, R. M., Lacourse, D. M., \& Crystal, J. D. (1998). Temporal search as a function of variability of interfood intervals. Journal of Experimental Psychology: Animal Behavior Processes, 24, 291-315.

Cooper, L. D. (1991). Temporal factors in classical conditioning. Learning and Motivation, 22, 129-152.

Davis, H., McIntire, R. W., \& Cohen, S. I. (1969). Fixed and variable duration warning stimuli and conditioned suppression. Journal of Psychology, 73, 19-25.

Delamater, A. R. (1995). Outcome-selective effects of intertrial reinforcement in a Pavlovian appetitive conditioning paradigm with rats. Animal Learning \& Behavior, 23, 31-39.

Desmond, J. E., \& Moore, J. W. (1988). Adaptive timing in neural networks: The conditioned response. Biological Cybernetics, 58, $405-415$.

Desmond, J. E., \& Moore, J. W. (1991). Altering the synchrony of stimulus trace processes: Tests of a neural-network model. Biological Cybernetics, 65, 161-169.

Egger, M. D., \& Miller, N. E. (1963). When is a reward reinforcing? An experimental study of the information hypothesis. Journal of Comparative and Physiological Psychology, 56, 132-137.

Evans, M., Hastings, N., \& Peacock, B. (1993). Statistical distributions. New York: Wiley.

Gallistel, R., \& Gibbon, J. (2000). Time, rate and conditioning. Psychological Review, 107, 289-344.

Gibbon, J. (1977). Scalar expectancy theory and Weber's law in animal timing. Psychological Review, 84, 279-325.

Gibbon, J., \& Balsam, P. (1981). Spreading association in time. In C. M. Locurto, H. S. Terrace, \& J. Gibbon (Eds.), Autoshaping and conditioning theory (pp. 219-254). New York: Academic Press.

Goddard, M. J., \& Jenkins, H. M. (1988). Blocking of a CS-US association by a US-US association. Journal of Experimental Psychology: Animal Behavior Processes, 14, 177-186.

Grossberg, S., \& Schmajuk, N. A. (1989). Neural dynamics of adaptive timing and temporal discrimination during associative learning. Neural Networks, 2, 79-102.

Holder, M. D., \& Roberts, S. (1985). Comparison of timing and classical conditioning. Journal of Experimental Psychology: Animal Behavior Processes, 11, 172-193.

Holland, P. C. (1998). Temporal control in Pavlovian occasion setting. Behavioural Processes, 44, 225-236.

Kamin, L. J. (1960). Acquisition of avoidance with a variable CS-US interval. Canadian Journal of Psychology, 14, 1-6.

Kamin, L. J. (1961). Apparent adaptation effects in the acquisition of a conditioned emotional response. Canadian Journal of Psychology, 15, 176-188.

Kamin, L. J. (1968). "Attention-like" processes in classical conditioning. In M. R. Jones (Ed.), Miami symposium on the prediction of behavior, 1967: Aversive stimulation (pp. 9-31). Coral Gables, FL: University of Miami Press.

Kamin, L. J. (1969). Predictability, surprise, attention, and conditioning. In B. Campbell \& R. Church (Eds.), Punishment and aversive behavior (pp. 279-298). New York: Appleton-CenturyCrofts.

Kehoe, E. J., Graham-Clarke, P., \& Schreurs, B. G. (1989). Temporal patterns of the rabbit's nictitating membrane response to compound and component stimuli under mixed CS-US intervals. Behavioral Neuroscience, 103, 283-295.

Kehoe, E. J., Schreurs, B. G., \& Amodei, N. (1981). Blocking acquisition of the rabbit's membrane response to serial conditioned stimuli. Learning and Motivation, 12, 92-108. 
Kimble, G. A., \& Dufort, R. H. (1956). The associative factor in eyelid conditioning. Journal of Experimental Psychology, 52, 386-391.

Kirkpatrick, K., \& Church, R. M. (1998). Are separate theories of conditioning and timing necessary? Behavioural Processes, 44, 163-182.

Kremer, E. F. (1971). Truly random and traditional control procedures in CER conditioning in the rat. Joumal of Comparative and Physiological Psychology, 76, 441-445.

LaBarbera, J. D., \& Church, R. M. (1974). Magnitude of fear as a function of the expected time to an aversive event. Animal Learning \& Behavior, 2, 199-202.

Leak, T. M., \& Gibbon, J. (1995). Simultaneous timing of multiple intervals: Implications of the scalar property. Journal of Experimental Psychology: Animal Behavior Processes, 21, 3-19.

Libby, M. E., \& Church, R. M. (1975). Fear gradients as a function of the temporal interval between signal and aversive event in the rat. Journal of Comparative and Physiological Psychology, 88, 911-916.

Lund, C. A. (1976). Effects of variations in the temporal distribution of reinforcements on interval schedule performance. Journal of the Experimental Analysis of Behavior, 26, 155-164.

Machado, A. (1997). Learning the temporal dynamics of behavior. Psychological Review, 104, 241-265.

Mackintosh, N. J. (1973). Stimulus selection: Learning to ignore stimuli that predict no change in reinforcement. In R. A. Hinde \& J. Stevenson-Hinde (Eds.), Constraints on learning (pp. 75100). New York: Academic Press.

Mackintosh, N. J. (1975). A theory of attention: Variations in the associability of stimuli with reinforcement. Psychological Review, 82, 276-298.

Matzel, L. D., Schachtman, T. R., \& Miller, R. R. (1988). Learned irrelevance exceeds the sum of CS-preexposure and USpreexposure deficits. Journal of Experimental Psychology: Animal Behavior Processes, 14, 311-319.

Meck, W. H., \& Church, R. M. (1984). Simultaneous temporal processing. Journal of Experimental Psychology: Animal Behavior Processes, 10, 1-29.

Millenson, J. R., Kehoe, E. J., \& Gormezano, I. (1977). Classical conditioning of the rabbit's nictitating membrane response under fixed and mixed CS-US intervals. Learning and Motivation, 8, 351-366.

Moore, J. W., \& Choi, J.-S. (1997). The TD model of classical conditioning: Response topography and brain implementation. In J. W. Donahoe \& V. P. Dorsel (Eds.), Neural-networks models of cognition (pp. 387-405). New York: Elsevier Science.
Pavlov, I. P. (1927). Conditioned reflexes. London: Oxford.

Pearce, J. M., \& Hall, G. (1980). A model for Pavlovian learning: Variations in the effectiveness of conditioned but not unconditioned stimuli. Psychological Review, 87, 532-552.

Randich, A.. \& Ross, R. T. (1985). Contextual stimuli mediate the effects of pre- and postexposure to the unconditioned stimulus on conditioned suppression. In P. D. Balsam \& A. Tomie (Eds.), Context and learning (pp. 105-132). Hillsdale, NJ: Erlbaum.

Rescorla, R. A. (1997). Summation: Assessment of a configural theory. Animal Learning \& Behavior, 25, 200-209.

Rescorla, R. A., \& Wagner, A. R. (1972). A theory of Pavlovian conditioning: Variations in the effectiveness of reinforcement and nonreinforcement. In A. H. Black \& W. F. Prokasy (Eds.), Classical conditioning: II. Theory and research (pp. 64-99). New York: Appleton-Century-Crofts.

Savastano, H. I., \& Miller, R. R. (1998). Time as content in Pavlovian conditioning. Behavioural Processes, 44, 147-162.

Schreurs, B. G., \& Gormezano, I. (1982). Classical conditioning of the rabbit's nictitating membrane response to CS compounds: Effects of prior single-stimulus conditioning. Bulletin of the Psychonomic Society, 19, 365-368.

Sutton, R. S., \& Barto, A. G. (1981). Toward a modern theory of adaptive networks: Expectation and prediction. Psychological Review, 88, 135-170.

Sutton, R. S., \& Barto, A. G. (1990). Time derivative models of Pavlovian reinforcement. In M. R. Gabriel \& J. W. Moore (Eds.), Learning and computational neuroscience: Foundations of adaptive networks (pp. 497-537). Cambridge, MA: MIT Press.

Tatham, T. A., \& Zurn, K. R. (1989). The Med-PC experimental apparatus programming system. Behavioral Research Methods, Instruments, and Computers, 21, 294-302.

Tomie, A. (1981). Effects of unpredictable food on the subsequent acquisition of autoshaping: Analysis of the context blocking hypothesis. In C. M. Locurto, H. S. Terrace, \& J. Gibbon (Eds.), Autoshaping and conditioning theory (pp. 181-215). New York: Academic Press.

Williams, D. A., \& LoLordo, V. M. (1995). Time cues block the CS, but the CS does not block time cues. Quarterly Journal of Experimental Psychology: Comparative and Physiological Psychology, 48B, 97-116.

Received May 10, 1999

Revision received November 19, 1999 Accepted December 7, 1999 